\title{
Determinaciones y relaciones de interacción en el triángulo constituido por preguntas, creatividad y aprendizaje
}

\section{Determinations and relationships of interaction in the triangle of questions, creativity and learning}

\author{
Romina Elisondo. \\ Consejo Nacional de Investigaciones Científicas y Técnicas (CONICET). \\ Universidad Nacional de Río Cuarto. Argentina. \\ relisondo@gmail.com \\ Danilo Donolo. \\ Consejo Nacional de Investigaciones Científicas y Técnicas (CONICET). \\ Universidad Nacional de Río Cuarto. Argentina. \\ donolo@gmail.com
}

\begin{abstract}
Resumen
El objetivo del ensayo es argumentar teórica y empíricamente acerca de las relaciones entre preguntas, creatividad y aprendizaje; y proponer orientaciones para construir contextos educativos creativos. Iniciamos el escrito presentando planteos teóricos referidos al enfoque problem finding y destacamos la importancia de las preguntas en la evaluación de la creatividad. Para construir las argumentaciones, recuperamos resultados de una investigación reciente titulada Evaluación de la creatividad: análisis de variables alternativas relacionadas con la forma y el contenido de las respuestas en el test CREA. Luego, nos referimos a la creatividad como componente indispensable de los aprendizajes y proponemos una perspectiva educativa en la cual las preguntas son el eje para la construcción de contextos creativos de enseñanza y aprendizaje. Destacamos el papel de las preguntas únicas e inesperadas como estrategias de enseñanza y de aprendizaje. La perspectiva educativa propuesta también reconoce las particularidades de los contextos tecnológicos actuales como espacios propicios para la interrogación. Asimismo, argumentamos acerca de los sustentos cognitivos, metacognitivos y emocionales de las preguntas en contextos educativos. Por último, planteamos consideraciones respecto de las relaciones entre preguntas, creatividad y aprendizajes en contextos mediados tecnológicamente.
\end{abstract}

\section{Palabras clave}

Descubrimiento de problemas; Interrogación, Aprendizaje creativo; Tecnologías de la Información y Comunicación.

\begin{abstract}
The objective is to argue about relationships between questions, creativity and learning and propose guidelines to build creative educational contexts. We submit theoretical approaches relating to problem finding and stress the importance of the questions in assessing creativity. We retrieve results of a recent study entitled Evaluation of creativity: analysis of alternative variables related to the form and content of the answers in the test CREA, in which we analyze 12,000 questions, build typologies and alternative scoring for CREA. Then, we refer to creativity as an essential component of learning and propose an educational perspective in which questions are the focus for the construction of creative teaching and learning contexts.
\end{abstract}


We emphasize the role of the unique and unexpected questions as strategies for teaching and learning. The educational perspective proposal also recognizes the particular current technological contexts as spaces for interrogation. Furthermore, we argue about the cognitive, metacognitive and emotional underpinnings of the questions in educational contexts. Finally, we propose considerations regarding relations between questions, creativity and learning in contexts of technological mediation.

\section{Keywords}

Problem finding; Questioning; Creative learning; Information and communication technologies.

\section{1.- Introducción}

El objetivo del ensayo es argumentar teórica y empíricamente acerca de las relaciones entre preguntas, creatividad y aprendizaje, triángulo favorable para crear contextos creativos de enseñanza y aprendizaje.

La metáfora ${ }^{1}$ del triángulo ya ha sido utilizada en diferentes contextos académicos y científicos, por ejemplo el triángulo didáctico (contenido, alumno y docente); el triángulo educativo (familia, escuela comunidad); el triángulo gobierno, ciencia-tecnología y estructura productiva de Jorge Sábato ${ }^{2}$, entre otros triángulos construidos teóricamente para explicar relaciones entre conceptos, sistemas o perspectivas. Los triángulos dan la idea de interactividad y de interrelaciones entre los elementos, tal vez por eso sean utilizados como metáforas para explicar teorías y perspectivas en diversos campos. En otro trabajo (Elisondo, 2016), hemos considerado a las metáforas como manifestaciones de la creatividad en las ciencias. En esta línea argumentativa, nos interesa pensar la educación desde la metáfora del triángulo interactivo.

La metáfora del triángulo nos convoca. Tres puntos nodales y tres relaciones entre ellos. Cada uno interactúa con los otros dos para atender una relación escolar, pedagógica si se quiere, y que puede ser extendida a otras situaciones de aprendizaje social, cultural y de la vida diaria. La necesidad de los tres vértices del triángulo se resume en las siguientes afirmaciones: sin preguntas no hay creatividad ni aprendizaje, sin creatividad tampoco hay nuevas preguntas ni aprendizajes y sin aprendizajes no hay creatividad ni nuevas preguntas. Creatividad, aprendizaje y preguntas forman un triángulo en el cual los tres lados son indispensables. Presentamos posibles relaciones entre los tres vértices o nodos del triángulo; y las argumentaciones las construimos a partir de estudios bibliográficos y

\footnotetext{
${ }^{1}$ La metáfora es una figura retórica por la cual se establece relación de correspondencia entre elementos, cualidades o semejanzas que existen entre ambas. En general hacemos usos poéticos de las metáforas, y en otros casos aprovechamos de su simplicidad para entender conceptos y tramas más complejas de alguna realidad o explicación. Son justamente los parecidos los que sirven a la definición de una metáfora; sin embargo, hay que estar atentos a las diferencias sutiles o explícitas que están latentes al momento del conocimiento de una situación o hecho. En nuestro caso recuperamos algunos ejemplos para mostrar que allí no está la innovación y proponemos otra versión simplificada que nos interesa mostrar: una relación interactiva entre, preguntas, creatividad y aprendizajes.

${ }^{2}$ https://es.wikipedia.org/wiki/Tri\%C3\%A1ngulo_de_Sabatohttp://www.redalyc.org/pdf/140/14003220.pdfhtt p://dx.doi.org/10.5209/rev__RCED.2011.v22.n1.1 entre otros
}

Determinaciones y relaciones de interacción en el triángulo constituido por preguntas, creatividad y aprendizaje. Romina Elisondo y Danilo Donolo.

Página 2 de 17 
empíricos donde observamos múltiples y complejas vinculaciones entre preguntas, creatividad y aprendizaje. Los emergentes del triángulo nos permiten pensar en alternativas para crear contextos educativos innovadores. En este sentido nos referimos a triángulos propicios como contextos favorables para la enseñanza, el aprendizaje y la innovación educativa.

Los triángulos que proponemos no se constituyen en el vacío, sino en contextos sociales más amplios caracterizados por el flujo dinámico de las informaciones y la constante interacción de los sujetos con artefactos tecnológicos de comunicación. Según Fainholc (2016), los vertiginosos cambios en las comunicaciones y las revoluciones tecnológicas permanentes generan un entorno disruptivo que interpela a las instituciones educativas respecto de los rumbos a seguir. Estos contextos configuran un marco particular para pensar las interacciones entre las preguntas, la creatividad y los aprendizajes. En esta línea, se asume una postura socio-cultural de la educación y la creatividad (Glaveanu, 2015) que considera las interacciones entre sujetos, lenguajes, artefactos e historias de las comunidades.

Para la construcción de las argumentaciones teóricas y empíricas se recuperan desarrollos y resultados de un estudio reciente en el que se analizan procedimientos de evaluación de capacidades creativas, específicamente desempeños de 4090 niños, adolescentes y adultos en el Test CREA $^{3}$ (Corbalán et al., 2015). El trabajo de investigación se tituló Evaluación de la creatividad: análisis de variables alternativas relacionadas con la forma y el contenido de las respuestas en el test CREA (Elisondo, 2015a). En la primera etapa de la investigación estudiamos la cantidad de preguntas formuladas por los participantes en el CREA y construimos baremos que atienden a diversidades socio-demográficas. Luego, analizamos el tipo de preguntas construidas por los participantes, se estudiaron 12399 interrogantes elaborados por niños/as, adolescentes y adultos ante los tres estímulos del CREA. A partir del análisis cualitativo, se elaboró un sistema alternativo de puntuación de los interrogantes considerando preguntas muy frecuentes, frecuentes y únicas. En la última etapa se analizaron relaciones entre el sistema tradicional que considera la cantidad de preguntas y el alternativo que atiende al tipo de interrogantes formulados. Entre los resultados más importantes, se hallaron correlaciones elevadas entre el procedimiento tradicional y el alternativo. "Si bien parece más simple y económico utilizar el sistema tradicional, el alternativo muestra interesantes datos para el análisis de casos, especialmente los que obtienen puntajes bajos según las pautas del manual” (Elisondo, 2015a: 168).

Tal vez, la relevancia del estudio no esté dada tanto por las metodologías, los resultados y los análisis, sino por el papel central que se les asigna a las preguntas en la creatividad. En la propuesta, las preguntas son objeto de análisis, interpelación y reflexión, miles de preguntas son leídas, analizadas, clasificadas y utilizadas para conocer acerca de las capacidades creativas de las personas. Quizás la importancia de la investigación radique en el supuesto básico que la sostiene: las preguntas son manifestaciones de creatividad, son la punta del iceberg de un complejo proceso de pensamiento y de vinculaciones entre conocimientos y esquemas diversos. Este supuesto general, recuperado de la mencionada investigación empírica (Elisondo, 2015a), sustenta los argumentos construidos en el presente ensayo.

\footnotetext{
${ }^{3}$ El Test CREA utiliza como procedimiento de medida de la creatividad la capacidad del sujeto para elaborar preguntas. La prueba consta de tres láminas estímulo (A, B y C) a partir de las cuales las personas deben formular la mayor cantidad de interrogantes posibles en cuatro minutos (Corbalán et al., 2015).
}

Determinaciones y relaciones de interacción en el triángulo constituido por preguntas, creatividad y aprendizaje. Romina Elisondo y Danilo Donolo.

Página 3 de 17 
Iniciamos el ensayo presentando planteos teóricos referidos al enfoque problem finding de estudio de la creatividad y destacamos la importancia de las preguntas en la evaluación de la creatividad. Luego, nos referimos a la creatividad como componente indispensable de los aprendizajes y proponemos una perspectiva educativa en la cual las preguntas son el eje para la construcción de contextos creativos de enseñanza y aprendizaje. Por último, planteamos consideraciones respecto de las relaciones entre preguntas, creatividad y aprendizajes.

\section{2.- Teorías de la creatividad}

El campo actual de investigación de la creatividad se caracteriza, entre otras cuestiones, por el desarrollo de diversas perspectivas, teorías y procedimientos metodológicos ${ }^{4}$. Precisamente, los avances en el campo están dados por los progresos logrados en estudios llevados a cabo desde diferentes enfoques, técnicas e instrumentos. Los estudios psicométricos iniciales, predominantes en la década de 1950, se han complejizado, los investigadores buscan realizar triangulaciones entre los test y otros instrumentos en poblaciones cada vez más diversas. A la vez, se han logrado significativos avances en los estudios experimentales, cognitivos, neuropsicológicos y computacionales, en parte, gracias a los avances tecnológicos que han permitido desarrollar estudios cada vez más sofisticados técnicamente para mayor número de casos y con variables más complejas que necesitan de información y datos más sensibles y sutiles para la realidad estudiada. Los estudios biográficos también han realizado aportes significativos al campo de la creatividad, ampliando perspectivas de análisis, casos y contextos de investigación. Actualmente, las perspectivas culturales (Glaveanu, 2015), buscan consolidarse en el campo, atendiendo a las influencias de los lenguajes, las culturas y las comunidades en los procesos creativos.

Sin embargo, no todas las perspectivas y metodologías se han desarrollado de igual manera en el campo de la creatividad. Haiying Long (2014) analizó artículos en revistas especializadas de creatividad, la investigadora observó que las metodologías cuantitativas, específicamente psicométricas y experimentales, son las que prevalecen en el campo actual. Asimismo, los test, los cuestionarios estandarizados y las técnicas de evaluación de productos y tareas, son los instrumentos que más utilizan los investigadores de los procesos creativos. En las investigaciones cualitativas, menos utilizadas entre los investigadores, preponderan los estudios de casos, las metodologías cualitativas básicas y la teoría fundada. En el estudio de Long (2014) se observaron pocas investigaciones que desarrollen diseños mixtos de integración de lógicas cuantitativas y cualitativas. Long señala interesantes líneas futuras de investigación: desarrollo de metodologías cualitativas que estudien la experiencia creativa de las personas en contextos cotidianos, realización de estudios en diferentes

\footnotetext{
${ }^{4}$ Mientras hablamos es esto, la vida creativa continúa en todos los ámbitos. A veces se carga a la educación que no ha progresado lo suficiente. Sin embargo, a poco de observar, los hechos nos dan algunos toques de realidad. Veamos sino la Universidad 42, que no tiene profesores, ni materias, ni asistencia a clases, casi ni edificios diría, y sin embargo promueve egresados con altísima capacidad de información, de resolución de problemas y de creatividad para hacerlo. Sus condiciones para el desempeño laboral son superlativas en un ambiente en el que se premie el trabajo y la iniciativa (http://www.bbc.com/mundo/noticias-36474360 universidad 42 sin profesores y mejor para la mente).
}

Determinaciones y relaciones de interacción en el triángulo constituido por preguntas, creatividad y aprendizaje. Romina Elisondo y Danilo Donolo.

Página 4 de 17 
contextos culturales, integración de diferentes perspectivas dentro del campo y comparaciones con otras áreas de la psicología y la educación ${ }^{5}$.

También podríamos definir al campo actual de investigación de la creatividad a partir de las teorías predominantes identificadas y descriptas por Kozbelt, Beghetto y Runco (2010). Los investigadores describen diez tipos de teorías que se diferencian según supuestos básicos subyacentes, foco de análisis, nivel de creatividad analizado y contexto conceptual de referencia. Las teorías psicométricas consideran que la creatividad puede ser medida a través de instrumentos estandarizados. Las teorías del desarrollo entienden que las personas despliegan la creatividad durante su vida a partir de las interacciones que se producen en diferentes contextos. Las teorías económicas analizan los procesos creativos desde la óptica del mercado, de los costos y beneficios de las actividades creativas. Las teorías de etapa o componenciales estudian los momentos y elementos de procesos creativos desde perspectivas lineales o recursivas. Las teorías cognitivas investigan los diferentes procesos de pensamiento que intervienen en la creatividad (combinación conceptual, expansión, metáforas, etc.). Las teorías centradas en la resolución de problemas estudian las soluciones creativas a tareas específicas, en cambio las teorías de formulación investigan los procedimientos vinculados a la generación de problemas e interrogantes. Las teorías evolucionistas consideran que las eminencias creativas surgen de procesos de evolución, generación y selección de ideas. Las teorías tipológicas pretenden identificar diferentes categorías de creadores. Las teorías sistemáticas explican los procesos creativos como interrelaciones complejas entre diferentes elementos ${ }^{6}$.

Para construir argumentaciones respecto de la importancia de las preguntas en la creatividad y la educación, nos interesan especialmente las teorías vinculadas al descubrimiento y la formulación de problemas. Desde estas teorías se destaca el papel de la formulación de problemas en los procesos creativos (Runco, 1994). Siguiendo estas perspectivas se han construido instrumentos de evaluación de la creatividad como el Test CREA (Corbalán et al., 2015) y el test de creatividad infantil TCI (Romo, AlfonsoBenlliure y Sanchez-Ruiz, 2016) que se basa en tareas de descubrimiento de problemas. Por eso, pensamos que las preguntas deben formar parte del triángulo necesario para la innovación educativa. Las preguntas son una parte esencial de la creatividad y también de los aprendizajes. A partir de las preguntas, los problemas, las cuestiones desconocidas es posible crear y recrear nuevos conocimientos.

\section{3.- Indicadores de capacidades creativas}

Nos preguntamos por las preguntas y las posibilidades que éstas ofrecen para evaluar capacidades creativas. No pretendemos en este apartado responder esta compleja pregunta, sino habilitar nuevos interrogantes y algunas respuestas que indican que formular problemas (preguntas) es indispensable en la creatividad y además, un buen indicador de capacidades creativas. Generar preguntas, cuestionar el mundo y lo establecido define a los

\footnotetext{
${ }^{5}$ Atendemos a esta consideración en diversos estudios sobre innovaciones educativas orientadas a la promoción de la creatividad, como por ejemplo, trabajos en museos (Elisondo y Melgar, 2015), propuestas de alfabetización informacional y creación de contextos inesperados de aprendizaje (Elisondo y Donolo, 2015).

${ }^{6}$ En los estudios de campo generalmente se integran algunas de las teorías descriptas. Por ejemplo, en las investigaciones presentadas en el libro Estudios de Creatividad. Las travesías de Alfonsina, de Astor, de Julios y de Marías, integramos teorías del desarrollo, psicométricas, cognitivas y sistémicas. El libro es de acceso abierto y está disponible en http://www.revistalatinacs.org/067/cuadernos/10CBA.pdf
}

Determinaciones y relaciones de interacción en el triángulo constituido por preguntas, creatividad y aprendizaje. Romina Elisondo y Danilo Donolo.

Página 5 de 17 
procesos creativos. Interrogantes y creatividad son interdependientes, no hay creatividad sin preguntas, ni preguntas sin creatividad. Las relaciones entre creatividad y preguntas se materializan en el proyecto CREA, es decir la iniciativa de evaluar inteligencia creativa tomando como indicador a la capacidad de la personas de hacer preguntas. El Test CREA Una medida cognitiva de la creatividad, emergente también del contexto iberoamericano y de propuestas teóricas destacadas en el campo de la creatividad como las teorías finding problems, propone un procedimiento novedoso y a la vez eficiente para evaluar inteligencia creativa: la formulación de interrogantes a partir de estímulos visuales ${ }^{7}$.

La simplicidad del CREA muchas veces hace dudar a los investigadores respecto de la validez y la confiabilidad del procedimiento (Martínez-Otero, 2005). Sin embargo, diversos estudios que hemos realizado aportan argumentos acerca de la pertinencia del indicador utilizado por el CREA. En el estudio Evaluation of creativity: analysis of alternative variables related to the form and content of the answers in the test CREA, hemos encontrado correlaciones elevadas y significativas entre las tres láminas en grupos de niños, adolescentes y adultos. Asimismo, observamos que los tres grupos formulan preguntas similares ante cada lámina, hallamos correlaciones elevadas entre el procedimiento tradicional del CREA y el alternativo, y asociaciones entre niveles de creatividad definidos según ambos procedimientos (Elisondo, 2015a).

Constantemente en nuestros estudios observamos que formular muchas preguntas se relaciona con participar en muchas actividades extracurriculares y de ocio. En diversas investigaciones, con grupos heterogéneos y en diferentes contextos hallamos que las personas que participan en más actividades en su vida diaria y académica, es decir son más abiertas a las experiencias, obtienen puntajes en el CREA significativamente superiores respecto de quienes no participan. También encontramos relaciones entre las puntuaciones en el CREA y las autovaloraciones de las personas respecto de sus capacidades creativas, quienes se consideran más creativos tienen puntajes significativamente superiores en el CREA. Asimismo, observamos interesantes correlaciones entre el CREA e instrumentos de evaluación de logros creativos (Elisondo y Donolo, 2014; 2016a y b).

Entonces, hacer preguntas parece ser un buen indicador de creatividad que se vincula con la apertura a la experiencia, las autopercepciones creativas y los logros creativos. Las personas que hacen más preguntas se consideran más creativas, participan en más actividades creativas y logran mejores desempeños en acciones concretas vinculadas a la creatividad en diferentes áreas de conocimiento. Hacer preguntas, encontrar problemas es

\footnotetext{
${ }^{7}$ Generalmente uno cree que tiene que aparecer la palabra pregunta o problema para que luego uno se aboque a solucionarlo... si tiene ganas por cierto. Dos ejemplos mostrarán la diversidad posible de formulaciones encubiertas que pueden aparecer... y es por eso que llamamos descubrir el problema, o formularse una pregunta. En el primer caso, a la Señora le diagnostican cáncer. Ella resume su existencia en una frase: Se arrepiente de haber sido ama de casa y haber dejado sus gustos sin realizar. Decide iniciar una nueva vida en lo que aprecia: la observación de las aves. Recorre el mundo cumpliendo su sueño.

http://internacional.elpais.com/internacional/2016/06/09/colombia/1465423864_988866.html Su iniciativa promueve las ganas de vivir ante toda desesperanza... (Ni la propuesta de Camus en su literatura puede reflejar más optimismo). Y murió en un accidente de automóvil habiéndose convertido en la mayor observadora de pájaros del mundo..., "tengo cáncer... no me voy a quedar en casa convaleciente".

El segundo caso, da respuesta a la pregunta tantas veces oída... sobre para qué sirve aprender o saber tal o cual cosa... lo hagamos exigente. Para qué sirven las matemáticas? http://one.elpais.com/tipo-te-convenceralas-matematicas-la-profesion-del-futuro/ los matemáticos hacen falta... las matemáticas son el motor de los próximos descubrimientos e innovaciones y serán necesarias para cualquier iniciativa que se desee concretar.
}

Determinaciones y relaciones de interacción en el triángulo constituido por preguntas, creatividad y aprendizaje. Romina Elisondo y Danilo Donolo.

Página 6 de 17 
esencial, en la creatividad tal como sostienen los investigadores de las perspectivas problem finding, es un buen indicador de capacidades creativas, como avala el CREA y también diversos estudios posteriores en los que se observan múltiples relaciones entre cantidad de preguntas, participación en actividades de ocio, apertura a la experiencia, autopercepciones y logros creativos.

El argumento que sigue tiene que ver con el aprendizaje, la observación está asociada al valor del conocimiento: saber sobre algo o saber dónde buscar son claves tanto para el aprendizaje como para la creatividad.

\section{4.- Componente creativo de los aprendizajes}

¿Puede el aprendizaje no ser creativo? Se preguntaba Zapata Ros (2002) en una interesante reflexión sobre creatividad, educación y aprendizaje. Desde nuestra perspectiva, la respuesta es NO. La creatividad juega un papel importante en todos los aprendizajes. Los procesos de construcción de conocimientos requieren preguntas e interpretaciones novedosas de la realidad en general, y de los contenidos educativos, en particular.

Para argumentar acerca del papel de la creatividad en los aprendizajes nos basamos en el Modelo de las Cuatro Creatividades propuesto por Kaufman y Beghetto (2009). Además de la creatividad con mayúsculas y creatividad con minúsculas, el modelo incorpora la mini creatividad y la pro- creatividad. La creatividad con mayúscula refiere a procesos creativos reconocidos socialmente por sus contribuciones "revolucionarias" a campos particulares de conocimiento. La creatividad con minúscula o creatividad cotidiana se vincula con expresión de la creatividad en la resolución de situaciones de la vida diaria. La minicreatividad implica interpretaciones nuevas y personales de experiencias, acciones y eventos. La mini-creatividad es inherente a todos los aprendizajes. La pro-creatividad es un tipo de creatividad que supone cierto nivel de conocimiento y experiencia en algún área específica. Las personas pro-creativas serían aquellas que disponen de una amplia formación y experiencia en un dominio específico pero aún no han llegado a ser creativos con mayúsculas, aunque tienen importantes chances de serlo.

Para nuestra argumentación nos interesa especialmente la mini-creatividad en tanto creatividad propia de todos los aprendizajes. Las interpretaciones novedosas sobre situaciones, objetos y relaciones, es decir las mini-creatividades siempre están presentes en los aprendizajes, independientemente de la complejidad de los mismos y de los contenidos a los que refieren. Por ejemplo, cuando un niño se da cuenta de que juntando las letras $\mathrm{S}$ e I se forma la palabra SI estamos ante la presencia de una interpretación novedosa para el sujeto que aprende. La mini-creatividad siempre es subjetiva y personal, no es novedoso para la mayoría de las personas sino solo para en el que ese momento experimenta esa nueva interpretación de la realidad. En este sentido, todos los aprendizajes tienen una cuota de creatividad.

Sin embargo, el aprendizaje no es solo una cuestión individual, sino que tal como propone Beghetto (2016), es un proceso intrasubjetivo e intersubjetivo que implica necesariamente relaciones con otras personas y sus respectivos puntos de $v^{2}$ sta $^{8}$. Las relaciones

\footnotetext{
${ }^{8}$ Ejemplos interesantemente variados se pueden encontrar en este link http://one.elpais.com/institutotecnologico-massachusetts-mit-mas-150-anos-inventado-futuro-la-humanidad/ inventando el futuro en la propuesta de ONE y MIT.
}

Determinaciones y relaciones de interacción en el triángulo constituido por preguntas, creatividad y aprendizaje. Romina Elisondo y Danilo Donolo.

Página 7 de 17 
interdependientes entre creatividad y aprendizaje articulan aspectos cognitivos intersubjetivos y vínculos intersubjetivos con otras personas. La concepción de aprendizaje creativo que sostenemos, en definitiva, integra perspectivas cognitivas y culturales del campo de investigación de la creatividad. El aprendizaje creativo supone procesos divergentes, alternativos y originales de compresión y pensamiento que no quedan solo en la "mente" de los sujetos sino que son puestos en juego y confrontado con perspectivas e ideas de otras personas. En ambos procesos, los de comprensión personal y los de aprendizaje con otros, las preguntas, las propias y las de los demás, posibilitan el aprendizaje creativo, sin ellas las nuevas perspectivas e ideas no emergen ni pueden ser confrontadas en grupo. Las preguntas, las que se hace el sujeto y las que hace a los demás, serían herramientas para el aprendizaje creativo.

\section{5.-Triángulos educativos propicios para la creatividad}

Encontrar problemas, formular preguntas constituyen una parte esencial de los procesos creativos y un indicador de capacidades creativas tal como plantean las teorías de descubrimiento de problemas y el test CREA. Entonces, tal vez formular preguntas sea una buena propuesta para construir contextos creativos de enseñanza y aprendizaje. Nos referimos a formular preguntas en sentido amplio, no solo a las tradicionales preguntas ¿cuánto es dos más dos? ¿Quién descubrió América?, sino, y especialmente, plantear interrogantes que promuevan aprendizajes creativos y comprensiones novedosas acerca de los objetos, los contenidos y las situaciones, es decir mini-creatividades. Interesa comprender a las preguntas como posibilidades de aprender creativamente, y no solo como procedimiento para la comprobación o evaluación de los aprendizajes. Para construir contextos creativos es indispensable habilitar espacios para preguntar, no basta con decir: ¿alguna pregunta?, sino que es necesario un clima de confianza y respeto donde todos, docentes y estudiantes, puedan exponer lo que no saben y lo que quieren saber. Las preguntas también evidencian conocimientos, siempre se pregunta sobre la base de algunos saberes previos. Proponemos una perspectiva generosa respecto de las preguntas, una mirada inclusiva que valore el desafío asumido por quien pregunta y los conocimientos y las emociones puestas en juego en el arte de preguntar. En los contextos educativos todas las preguntas pueden ser relevantes, al menos, indican de alguna manera creatividad, tal como hemos argumentado en párrafos anteriores ${ }^{9}$.

\section{La interrogación como estrategia de enseñanza y aprendizaje}

Preguntar siempre supone un conocimiento, en realidad varios conocimientos puestos en juego, un interés manifiesto por conocer algo y por vincularse con el entorno. Las preguntas no surgen en el vacío sino que se sustentan en informaciones y experiencias emergentes de los entornos. Entre las preguntas y los conocimientos se producen relaciones dialécticas, los conocimientos activan preguntas y las preguntas permiten construir nuevos conocimientos. Desde diversas perspectivas puede comprenderse la importancia de las preguntas en los aprendizajes, las teorías psicoanalíticas, por ejemplo, consideran que el deseo de aprender depende de preguntas respecto de la sexualidad y las relaciones humanas (Tenorio y

\footnotetext{
${ }^{9}$ Preguntas e ideas que inspiran. http://www.lanacion.com.ar/1894782-ideas-que-inspiran-joshua-prager-unescritor-tras-la-sabiduria-de-cada-edad-de-la-vida TED en ideas que inspiran, 100 años de la sabiduría de un escritor.
}

Determinaciones y relaciones de interacción en el triángulo constituido por preguntas, creatividad y aprendizaje. Romina Elisondo y Danilo Donolo.

Página 8 de 17 
Jacobo, 2013). Desde las didácticas y los interesados en la enseñanza, se promueve el uso de las preguntas como recurso pedagógico y también se estimula a los estudiantes a formular interrogantes como estrategia de aprendizaje. Los investigadores destacan la importancia de promover procesos de formulación de preguntas en las aulas como forma de propiciar la comprensión lectora y el aprendizaje significativo (Márquez, Pujol y Bonil, 2005; Silvestri, 2009; Tort, 2005) Entre los resultados más importantes en el campo del uso de las preguntas en las enseñanza, se han visualizado diferencias en las tipologías de interrogantes según rendimiento académico. Los estudiantes con mejor rendimiento formulan más preguntas inferenciales que los de menor rendimiento (Mazzitelli, Maturano y Macias, 2009). En el caso del nivel educativo, no se observaron diferencias en el tipo y la complejidad de las preguntas elaboradas por estudiantes de diferentes grados (Colmenares et al., 2015). Desde las pedagogías críticas, las preguntas tienen un papel clave en la educación. Freire y Faúndez (1986) proponen una pedagogía de la pregunta que enseñe a preguntar, que estimule la capacidad de asombro y la curiosidad de docentes y estudiantes. La pedagogía de la pregunta permite a los estudiantes proponer y demandar conocimientos. La pedagogía de la pregunta se basa en el diálogo, el respecto y el aprendizaje con otros.

En los contextos educativos es relevante preguntarse por los interrogantes que despiertan los conocimientos, los objetos, las relaciones y los recursos que circulan en las instituciones educativas. En las aulas, interesan las preguntas, aquellas que abren caminos a la imaginación, la innovación y la transformación, y también las que cuestionan las normas, las que proponen transgresiones. Son relevantes para la creatividad las preguntas difíciles de responder y las personas a quienes no les satisface ninguna respuesta y buscan alternativas para que la pregunta no pueda ser respondida completamente, y así, nunca terminar la búsqueda de conocimientos. Divergencia inherente a la creatividad está representada por las preguntas: múltiples alternativas, diversas perspectivas, infinitas posibilidades ${ }^{10}$. Generar espacios genuinos para formular y responder preguntas de diferente complejidad es una intervención pedagógica necesaria para la construcción de contextos creativos de enseñanza y aprendizaje. Las preguntas suponen relaciones con el mundo y los conocimientos, deseos de conocer y comunicarse con otros. Las preguntas abren los canales necesarios para el dialogo, el aprendizaje y la creatividad.

\section{Preguntas, posibilidades de pensamiento y mini-creatividad}

Según Craft (2013), las posibilidades de pensamiento son infinitas, entonces, en los contextos educativos es necesario generar espacios donde esas posibilidades puedan expandirse a partir de preguntas y problemas. Cremin et al.,(2015) señalan que la curiosidad y la formulación de preguntas, tanto en docentes como en estudiantes, tiene un impacto positivo en la educación creativa. Los autores destacan la importancia de las preguntas abiertas, del diálogo y la colaboración entre compañeros y docentes y de los factores emocionales y motivacionales en la enseñanza creativa. Los investigadores plantean a los estudiantes, en el marco de investigaciones cualitativas en el aula, problemas y situaciones a partir de la pregunta ¿qué pasaría si...? (what if?). Entre los resultados más

\footnotetext{
${ }^{10}$ Innovar es una forma de crear. ¿Cómo se hace? http://www.lanacion.com.ar/1906922-consejos-parainnovar-de-dos-mentes-brillantes para mentes brillantes e innovación.
}

Determinaciones y relaciones de interacción en el triángulo constituido por preguntas, creatividad y aprendizaje. Romina Elisondo y Danilo Donolo.

Página 9 de 17 
importantes, han observado multiplicidad de respuestas y de intercambios entre los estudiantes que dan cuenta de las infinitas posibilidades de pensamiento ${ }^{11}$.

Las preguntas también son importantes para la mini-creatividad (Beghetto y Kaufman, 2014), es decir para a creatividad que compone todos los aprendizajes ya que suponen nuevos significados e interpretaciones sobre la realidad, los objetos, las situaciones y las relaciones entre conocimientos. Según los autores, las prácticas docentes que favorecen la creatividad son aquellas que explícitamente promueven el pensamiento creativo, la motivación intrínseca y la imaginación, ofrecen oportunidades de elección y descubrimiento y establecen un ambiente propicio para el aprendizaje. Asimismo, estimulan la creatividad aquellas prácticas docentes que interpelan a los estudiantes a generar múltiples ideas, redefinir problemas, usar analogías y evaluar perspectivas y productos, asumiendo la responsabilidad de los propios aprendizajes y desarrollando estrategias metacognitivas. Los docentes que promueven la creatividad son aquellos que estimulan a los estudiantes con mensajes motivadores, promueven la curiosidad, la autoexpresión y la asunción de riesgos y ofrecen devoluciones o feedbacks ajustados a los procesos de creación de conocimientos.

Según Beghetto (2013), promover la creatividad en la clase supone trabajar con micromomentos creativos, momentos donde emergen preguntas y respuestas inesperadas. Desde la perspectiva del autor y los estudios que ha desarrollado, los micromomentos creativos rompen con lo esperable en la clase y abren interesantes espacios para las preguntas, las interpretaciones novedosas $\mathrm{y}$ las respuestas originales. En los micromomentos creativos es importante que los docentes desarrollen intervenciones adecuadas que promuevan ideas novedosas e interacciones entre los estudiantes para evaluar y complejizar las preguntas y las respuestas emergentes. El diálogo y la discusión entre los actores educativos es el contexto más propicio para el desarrollo de la creatividad en las clases, independientemente de las áreas de conocimientos que se trabajen y los grupos de estudiantes.

El modelo de aprendizaje de Beghetto (2016) enfatiza aspectos intersubjetivos, interpretaciones novedosas de los sujetos, e intersubjetivo, interacciones con otros respecto de dichas interpretaciones o mini-creatividades. En la etapa intrasubjetiva, el modelo propuesto incluye los siguientes momentos: consideración por parte del sujeto de estímulos óptimos discrepantes (propuestos por el docente), combinación de estímulos nuevos con conocimientos existentes, interpretaciones nuevas personalmente significativas, nuevas comprensiones subjetivas respecto de los objetos. En la etapa intersubjetiva, los momentos principales son: oportunidad de expresión y de compartir conocimientos con otros, evaluación de ideas y discrepancias con otros, apoyo del grupo para comprender conceptos aparentemente incompatibles, determinación de compatibilidad de conceptos discrepantes y reconocimiento de la contribución creativa. Según Beghetto, las aulas que apoyan el

\footnotetext{
${ }^{11}$ Los dos links muestras formas distintas de ser creativos e innovar. El premio hace evidente el fin de un trabajo prometedor; en tanto que la reserva, resguarda el descubrimiento. http://www.ieco.clarin.com/campusieco/equipo-UDESA-competira-desafio-LOreal_0_1591040988.html premiados por L`Oreal por un trabajo de la universidad de San Andrés. http://www.bbc.com/mundo/noticias/2016/06/160606_brasil_ave_desaparecida_am el pájaro de ojos azules reapareció... ¿innovación, creación, descubrimiento, hallazgo fortuito?
}

Determinaciones y relaciones de interacción en el triángulo constituido por preguntas, creatividad y aprendizaje. Romina Elisondo y Danilo Donolo.

Página 10 de 17 
aprendizaje creativo ofrecen oportunidades para los estudiantes y profesores de aprender y enseñar en colaboración.

Claramente las propuestas de aprendizaje creativo se sustentan en perspectivas socioculturales de la educación, el aprendizaje y la creatividad. Los otros y los artefactos culturales tienen un papel destacado en las posibilidades de aprender y desarrollar la creatividad. En esta línea argumentativa, consideramos relevante atender a los artefactos tecnológicos como mediadores del aprendizaje colaborativo y la creatividad.

\section{Preguntas en contextos tecnológicos}

Desde perspectivas educativas socioculturales, el aprendizaje depende de interacciones con artefactos de la cultura. La inteligencia (Perkins, 2001) y la creatividad (Romero, 2010) están distribuidas entre las personas y los objetos. En el contexto actual, los artefactos tecnológicos ofrecen ilimitadas posibilidades de aprender, formular preguntas y buscar respuestas. Los artefactos tecnológicos ofrecen infinidad de conocimientos, informaciones y procedimientos, recurso necesarios para la creatividad. Las tecnologías también habilitan espacios nuevos de aprendizaje, juego e interacción social. Los tiempos y los lugares se complejizan, las realidades parecen múltiples (aumentadas, virtuales, etc.) y las formas de acceder a los conocimientos son impredecibles (González, 2014). En este complejo contexto, las preguntas y las respuestas, es decir los procesos creativos, fluyen sin límites. Las innovaciones educativas con mediaciones tecnológicas constituyen ambientes disruptivos propicios para la creatividad (Chibas, Borroto y Almeida, 2014; Steinbeck, 2011). Rompen con las rutinas, incorporan elementos inesperados y expanden las posibilidades de aprender y de hacerlo con otras personas. Las tecnologías generan espacios indisciplinados, ilimitados e inesperados para aprender, contextos propicios para la creatividad (Elisondo, 2015b).

Los planteos actuales de la educación expandida (Carrasco, Baldivieso y Di Lorenzo, 2016; Díaz y Freire, 2012; Fernández Rodríguez y Anguita Martínez, 2015; Neri y Fernández Zalazar, 2015) dan cuenta de las potencialidades de las tecnologías para transformar la educación, ampliándola y reconociendo tiempos y presencialidades diversas en los procesos de enseñanza y aprendizaje. Los procesos educativos pueden expandirse por múltiples y diversos espacios presenciales y virtuales. Expandir la educación es ampliar las posibilidades de interacciones entre sujetos y conocimientos mediados por tecnologías, lenguajes y herramientas diversas. Expandir la educación es transalfabetizar (Ipri, 2010), integrar alfabetizaciones y procesos de lectura crítica, producción y comunicación de información. No solo alfabetizar consumidores de información, sino educar personas con capacidades de producir, crear contenidos, comunicarlos y compartirlos con los demás. La producción de contenidos es el aspecto más creativo de la transalfabetización en tanto supone procesos de creación de nuevos conocimientos compartidos en comunidades y redes $^{12}$.

Preguntas y metacognición

Una buena pregunta es ¿qué es una pregunta? Preguntar es un proceso complejo que implica comprender metacognitivamente qué es construir una pregunta. Hemos observado

\footnotetext{
${ }^{12}$ Ver el link en relación con el argumento: http://www.lanacion.com.ar/1898606-la-regla-de-las-10000-horasmitos-y-verdades-sobre-el-conocimiento-y-el-talento la ley de las 10000 horas talento y capacitación.
}

Determinaciones y relaciones de interacción en el triángulo constituido por preguntas, creatividad y aprendizaje. Romina Elisondo y Danilo Donolo.

Página 11 de 17 
que los niños pequeños, menores de 7 años, a pesar de realizar constantemente múltiples y complejas preguntas, tienen dificultades cuando se les solicita que formulen una pregunta sobre una imagen. Observamos esta dificultad ante la consigna del Test CREA, la mayoría de los niños pequeños realizan descripciones de las imágenes y no preguntas sobre lo que observan en ellas (Elisondo, 2015a). En algunos grupos de adolescentes y adultos también observamos ciertas dificultades para formular preguntas en las etapas iniciales de la prueba, en los primeros momentos luego de la consigna. Posteriormente, pasados algunos segundos, comienzan a formular interrogantes. Algunas personas solicitan explicaciones adicionales respeto de la consigna del instrumento. Hacer preguntas no parece ser una actividad tan sencilla ni tan frecuente, al menos para un test. También hemos solicitado a estudiantes universitarios en contextos de evaluación que formulen preguntas respecto de los contenidos curriculares, esta consigna les genera sorpresa e incertidumbre en un primer momento, pareciera que en un examen solo el docente puede realizar preguntas. Hacer preguntas supone conocimientos metacognitivos respecto de qué es una pregunta, cuál es su estructura y cómo formularla.

La metacognición juega un papel importante en la formulación de preguntas en particular, y en la creatividad en general (Kaufman, Beghetto y Watson, 2015). Es importante en los contextos educativos propiciar espacios para formular preguntas y también para reflexionar metacognitivamente acerca de los procesos de construcción de interrogantes. Asimismo es interesante reflexionar sobre la complejidad de los diferentes tipos de preguntas y de los esquemas cognitivos y metacognitivos que subyacen a cada nueva cuestión.

Para construir contextos creativos de enseñanza y aprendizaje interesan las preguntas que formulan los alumnos pero también las construidas por los docentes. Varios autores (Márquez, Pujol y Bonil, 2005; Silvestri, 2009; Tort, 2005; Zuleta Araújo, 2005) destacan la importancia pedagógica de las preguntas en el aula y sus potencialidades para la promoción del aprendizaje. Interesan también las preguntas que formulan los docentes, no todas tienen el mismo nivel de complejidad ni promueven los mismos procesos cognitivos en los estudiantes. Tornero et al., (2015) señalan que es posible clasificar a las preguntas de los docentes en cerradas, interrogantes que tienen un número limitado de respuestas posibles, o abiertas, cuestiones que habilitan múltiples respuestas y demandan mayores grados de elaboración y abstracción. Las preguntas también pueden clasificarse en convergentes y divergentes, mientras que las primeras promueven soluciones tradicionales y requieren de la aplicación de un proceso ya existente como respuesta, las divergentes demandan procesos de razonamiento inéditos, dejan abiertas una multiplicidad de opciones de respuesta. Asimismo, las preguntas pueden catalogase según el nivel de abstracción que demandan: memoria, comprensión, interpretación, aplicación, análisis, síntesis y evaluación. Los autores proponen una clasificación de las preguntas de los docentes según nivel de complejidad y definen las siguientes categorías y subcategorías: recuperar (reconocer, reproducir, nombres, describir), comprender (exponer, secuencias, definir, sustituir), aplicar (ejecutar, transferir, hacer hipótesis), analizar (distinguir, explicar, comparar, categorizar, sintetizar), evaluar (juzgar, justificar) y crear (Tornero et al., 2015). Para la construcción de contextos creativos de enseñanza y aprendizaje resultan propicias las preguntas de los docentes que son abiertas, divergentes y complejas, es decir aquellas que suponen relaciones entre conocimientos, valoraciones y producciones de nuevos conocimientos y problemas. Asimismo, las preguntas que promueven diferentes procesos cognitivos, vínculos entre conocimientos diversos y múltiples respuestas parecen más favorables para la creatividad. Según nuestra perspectiva, para crear contextos innovadores

Determinaciones y relaciones de interacción en el triángulo constituido por preguntas, creatividad y aprendizaje. Romina Elisondo y Danilo Donolo.

Página 12 de 17 
es necesario reflexionar metacognitivamente respecto de las preguntas de los estudiantes y de los docentes. Resultan interesantes las preguntas y las respuestas inesperadas, de docentes y estudiantes, es decir aquellos interrogantes las que crean micromomentos propicios para la creatividad en las clases.

\section{6.- Consideraciones finales}

Las preguntas forman parte de la creatividad y a la vez, esta es parte constitutiva del aprendizaje. Entonces, serán propicios para el aprendizaje aquellos contextos educativos que promuevan el uso de preguntas como estrategia de enseñanza y de aprendizaje. Las teorías de la creatividad vinculadas a la formulación de problemas y las perspectivas sobre pensamiento divergente sustentan las consideraciones realizadas en el presente escrito. Los resultados de una investigación previa sobre formulación de preguntas y medición de la creatividad (Elisondo, 2015a) sustentan empíricamente las argumentaciones presentadas a lo largo del ensayo. En esa investigación se destacó la importancia del procedimiento de formular preguntas para la medición de la creatividad y de la diversidad de esquemas cognitivos incluidos en dicho procedimiento.

Las preguntas requieren de conocimientos y a la vez de deseos de conocer. Preguntar es construir un vínculo entre conocimientos previos y nuevos, preguntar es interactuar con otros y con los objetos de la cultura. Preguntar, en definitiva es una acción socio-cultural vinculada con el conocimiento. Claro está que existen diferentes tipos de preguntas y procesos cognitivos implicados y promovidos a partir de los interrogantes. Aunque todas las preguntas tienen una potencialidad educativa, interesan especialmente para los contextos creativos aquellas que refieren a contenidos alejados, las que son inesperadas e inusuales y las que suponen inferencias. Son bienvenidas las preguntas de los docentes y de los estudiantes que permiten crear nuevas relaciones entre conocimientos. Las respuestas que habilitan las preguntas también son importantes, para el aprendizaje y la creatividad aquellos interrogantes divergentes que posibilitan múltiples alternativas y soluciones resultan más propicios.

Sin embargo, preguntar no es fácil, tal como lo visualizamos permanentemente en nuestras investigaciones sobre creatividad y en las prácticas educativas. Preguntar requiere de sustentos cognitivos y metacognitivos, y también de contextos emocionales favorables. Preguntar es asumir riesgos, reconocer ignorancias y demostrar deseo de saber. En las aulas es necesario preguntar y enseñar a preguntar, a construir diferentes tipos de interrogantes que promuevan la comprensión y el aprendizaje. Asimismo, es indispensable construir contextos educativos con espacios para las preguntas y las respuestas complejas, inesperadas e inusuales.

Las mediaciones tecnológicas también generan contextos propicios para el cuestionamiento y la formulación de interrogantes. El acceso a múltiples y diversos contenidos, las posibilidades infinitas de interacciones y uso de lenguajes y estéticas alternativas configuran un campo fértil para las preguntas y la creación de conocimientos. Sin embargo, tal como sostiene Fainholc (2016), las tecnologías también pueden generar paradojas, contradicciones y desigualdades. Las tecnologías en educación plantean complejos enigmas, los impactos pueden ser positivos o negativos según las decisiones políticas y sociales que se tomen. "El uso interactivo de las redes, defenderá políticas de apertura horizontal, o puntos de vista defensores del statu quo? La imagen democrática e inclusiva de las TIC, ¿ocultará las inequidades y la e-exclusión social (etnias, género, nivel

Determinaciones y relaciones de interacción en el triángulo constituido por preguntas, creatividad y aprendizaje. Romina Elisondo y Danilo Donolo.

Página 13 de 17 
socioeconómico, geografía, edad, etc.)?" (Fainholc, 2016: 18). Los enigmas que plantea Fainholc al finalizar su escrito reflejan los grandes desafíos de las tecnologías y sus posibles usos e impactos en contextos sociales diversos.

¿Se anima Ud. a preguntar? Esperamos las interacciones propicias, los comentarios agudos y los señalamientos de incongruencias en relisondo@gmail.com ${ }^{13}$

Presentación del artículo: 30 de junio de 2016.

Fecha de aprobación: 19 de octubre de 2016.

Fecha de publicación: 15 de noviembre de 2016.

Elisondo, R. y D. Donolo (2016). Determinaciones y relaciones de causalidad e interacción en el triángulo constituido por preguntas, creatividad y aprendizaje. RED. Revista de Educación a Distancia, 51. Consultado el (dd/mm/aaaa) en http://www.um.es/ead/red/51

\section{Referencias bibliográficas}

Beghetto, R. (2016). Creative Learning: A Fresh Look. Journal of Cognitive Education and Psychology, 15 (1). Recuperado 30 de junio de 2016 de www.ronaldbeghetto.com/s/BeghettoJCEP_2016-uncorrected-proof-1tby.pdf

Beghetto, R. (2013). Nurturing Creativity in the Micro-moments of the Classroom. En Kim, K., Kaufman, J., Baer, J. y B. Sriraman. Creatively Gifted Students are not like Other Gifted Students. pp 3-15. Rotterdam: Sense Publishers.

Beghetto, R. y Kaufman, J. (2014). Classroom contexts for creativity. High Ability Studies, 25(1), 53-69.

Carrasco, S., Baldivieso, S. y Di Lorenzo, L. (2016). Formación en investigación educativa en la sociedad digital. Una experiencia innovadora de enseñanza en el nivel superior en el contexto latinoamericano. RED. Revista de Educación a Distancia. 48(6).

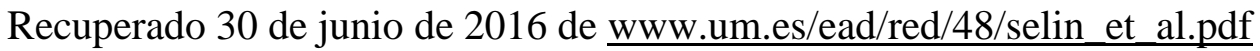

Chibas, F., Borroto, G. y Almeida, F. (2014). Gestión de la creatividad en entornos virtuales de aprendizaje colaborativos: un proyecto corporativo de EAD Comunicar, 43, 143-151. Recuperado 30 de junio de 2016 de http://dx.doi.org/10.3916/C43-2014$\underline{14}$

\footnotetext{
${ }^{13}$ Mientras va pensando las preguntas a hacernos vea los siguientes links: http://www.clarin.com/zona/desafiochicos-digitales-Argentina-preparada_0_1580842022.html las futuro. http://brainiq.noticiasinteligente.net/?t202id=4442\&c2=img1Nueva\&t202ref=social\%20and\%20trending\%20-\%20below\%20article\&t202kw=27791 la pastilla de la inteligencia...http://www.bbc.com/mundo/noticias/2016/05/160518_finde_tecnologia_cultura_em_forster_ho wards_end_internet_la_maquina_se_detiene_lb novela que predice en 1910 la creación de internet.
} 
Colmenares, D., Vergara, J., Rosales, S. y Carrascal, O. (2015). Grado de complejidad en la elaboración de preguntas por los alumnos a partir del análisis de un texto de divulgación científica. CITECSA, 5(9), 25-37.

Corbalán, J., Martínez, F., Donolo, D., Alonso, C., Tejerina, M. y Limiñana, M. (2015). CREA. Inteligencia Creativa. Una medida Cognitiva de la Creatividad. Madrid: TEA Ediciones. Segunda Edición.

Craft, A. (2013). Childhood, possibility thinking and wise, humanizing educational futures. International Journal of Educational Research, 61, 126-134.

Cremin, T., Glauert, E., Craft, A., Compton, A. y Styliandou, F. (2015). Creative little scientists: exploring pedagogical synergies between inquiry-based and creative approaches in Early Years science. Education, 43(4), 404-419

Elisondo, R. (2016). Creatividad y Ciencias Un estudio biográfico de científicos argentinos. Ciencia, Docencia y Tecnología, 27(52). Recuperado 30 de junio de 2016 de http://www.pcient.uner.edu.ar/index.php/cdyt/article/view/125/203

Elisondo, R. (2015a). Evaluación de la creatividad: análisis de variables alternativas relacionadas con la forma y el contenido de las respuestas en el test CREA. Tesis de Doctorado. Universidad de Murcia. Recuperado 30 de junio de 2016 de https://digitum.um.es/jspui/handle/10201/46976

Elisondo, R. (2015b). La creatividad como perspectiva educativa. Cinco ideas para pensar los contextos creativos de enseñanza y aprendizaje. Revista Actualidades Investigativas en Educación, 15(3) Recuperado 30 de junio de 2016 de http://revistas.ucr.ac.cr/index.php/aie/article/view/20904

Elisondo, R. y Donolo, D. (2014). Interculturalidad, apertura a experiencias y creatividad Aportes para una educación alternativa. Red Revista de Educación a Distancia, 41, 119. Recuperado 30 de junio de 2016 de http://www.redalyc.org/pdf/547/54731315004.pdf

Elisondo, R. y Donolo, D. (2015) Invitaciones, desafíos y apuestas. contextos para la creatividad. Revista electrónica de investigación, docencia y creatividad, 4, 11-19.

Elisondo, R. y Donolo, D. (2016a). Construcción y análisis de las propiedades psicométricas del Cuestionario de Acciones Creativas en población argentina. PSIENCIA. Revista Latinoamericana de Ciencia Psicológica, 8. doi: 10.5872/psiencia/8.1.42.

Elisondo, R. y Donolo, D. (2016b). Creativity, Involvement, and Leisure Time:The More, the Better. Business creativity and the creative economy, 2(1), 16-23.

Elisondo, R. y F. Melgar (2015) Museos y la Internet: contextos para la innovación. Revista Innovación educativa, 68: 17-32.

Fainholc, B. (2016). Presente y futuro latinoamericano de la enseñanza y el aprendizaje en entornos virtuales referidos a educación universitaria. RED. Revista de Educación a Distancia, 48(2). Recuperado 30 de junio de 2016 de http://www.um.es/ead/red/48

Fernández Rodríguez, E. y Anguita Martínez, R. (2015). Aprendizajes invisibles en contextos de educación expandida. Retos y oportunidades en la sociedad

Determinaciones y relaciones de interacción en el triángulo constituido por preguntas, creatividad y aprendizaje. Romina Elisondo y Danilo Donolo.

Página 15 de 17 
hiperconectada. Profesorado: Revista de curriculum y formación del profesorado, 19, $1-10$

Freire, P. y Faúndez, A. (1986). Por una pedagogía de la pregunta. Buenos Aires: Siglo XXI Editores.

Glaveanu, V. P. (2015). Creativity as a sociocultural act. The Journal of Creative Behavior, 49(3), 165-180.

González, C. (2014). Estrategias para trabajar la creatividad en la Educación Superior: pensamiento de diseño, aprendizaje basado en juegos y en proyectos. RED, Revista de Educación a Distancia, 40. Recuperado 30 de junio de 2016 de http://www.um.es/ead/red/40/

Ipri, T. (2010).Introducing transliteracy. What does it mean to academic libraries? College \& Research Libraries News, 71(10), 532-567.

Kaufman, J. y Beghetto, R. (2009). Beyond Big and Little: The Four C Model of Creativity. Review of General Psychology, 13 (1): 1-12.

Kaufman, J. Beghetto, R. y C. Watson (2015). Creative metacognition and self-ratings of creative performance: A 4-C perspective. Learning and Individual Differences, Recuperado 30 de junio de 2016 de http://dx.doi.org/10.1016/j.lindif.2015.05.004

Kozbelt, A.; Begheto, R.; M, Runco (2010). Theories of Creativity, (pp. 20-47). En Kaufman y Sternberg, The Cambridge Handbook of Creativity. Cambridge: Cambridge Press.

Long, H. (2014). An Empirical Review of Research Methodologies and Methods in Creativity Studies (2003-2012). Creativity Research Journal, 26(4), 427-438.

Márquez, C., Pujol, R. y Bonil, J. (2005). Las preguntas mediadoras como recursos para favorecer la construcción de modelos científicos complejos. Enseñanza de las Ciencias, (Extra), 1-5.

Martínez-Otero, V. (2005) Rumbos y desafíos en Psicopedagogía de la Creatividad. Revista Complutense de Educación, 16 (1),169-181.

Mazzitelli, C. A., Maturano, C. y Macías, A. (2009). Análisis de las preguntas que formulan los alumnos a partir de la lectura de un texto de Ciencias. REEC: Revista electrónica de enseñanza de las ciencias, 8(1), 3: 45-57.

Neri, C. y Fernández Zalazar, D. (2015). Apuntes para la revisión teórica de las TIC en el ámbito de la educación superior. RED. Revista de Educación a Distancia, 47(3). Recuperado 30 de junio de 2016 de http://www.um.es/ead/red/47

Perkins, D. (2001). La persona- más: una visión distribuida del pensamiento y el aprendizaje. En Salomon, G. (Comp.). Cogniciones distribuidas. Consideraciones psicológicas y educativas. Buenos Aires: Amorrortu. pp. 126-152.

Romero J. (2010). Creatividad distribuida y otros apoyos para la educación creadora. Pulso, $33,87-107$.

Romo, M., Alfonso-Benlliure, V. y Sanchez-Ruiz, M. (2016) El test de creatividad infantil(TCI):evaluando la creatividad mediante una tarea de encontrar problemas.

Determinaciones y relaciones de interacción en el triángulo constituido por preguntas, creatividad y aprendizaje. Romina Elisondo y Danilo Donolo.

Página 16 de 17 
Psicología Educativa. Recuperado 30 de junio de 2016 de http://www.sciencedirect.com/science/article/pii/S1135755X16000099

Runco, M. A. (1994). Problem finding, problem solving, and creativity. Greenwood: Publishing Group.

Silvestri, Adriana. (2006). La formulación de preguntas para la comprensión de textos: Estudio experimental. Revista signos, 39(62), 493-510.

Steinbeck, R. (2011). El «design thinking» como estrategia de creatividad en la distanciaComunicar, 37, 27-35.

Tenorio, B. y Jacobo, M. (2013) El deseo de saber en la infancia. Revista de Educación y Desarrollo, 24: 23- 28.

Tornero B., Ramaciotti A., Truffello A. y Valenzuela F. (2015). Nivel cognitivo de las preguntas que formulan las educadoras de párvulos. Educ, 18 (2), 261-283.

Tort, M. R. (2005). Las preguntas en el proceso de enseñanza aprendizaje de las ciencias. Educar, 25, 73-80.

Zapata Ros, M. (2002). ¿Puede el aprendizaje no ser creativo? RED. Revista de Educación a Distancia, 4, 1-5. Recuperado 30 de junio de 2016 de www.um.es/ead/red/4/creativ.pdf

Zuleta Araújo, O. (2005). La pedagogía de la pregunta, una contribución para el aprendizaje. Revista electrónica Educere, 9 (28), 115-119.

Determinaciones y relaciones de interacción en el triángulo constituido por preguntas, creatividad y aprendizaje. Romina Elisondo y Danilo Donolo. 